\title{
Controlling open quantum systems: Tools, achievements, and limitations
}

\author{
Christiane P. Koch ${ }^{1, *}$ \\ ${ }^{1}$ Theoretische Physik, Universität Kassel, Heinrich-Plett-Str. 40, 34132 Kassel, Germany
}

(Dated: March 31, 2016)

\begin{abstract}
The advent of quantum devices, which exploit the two essential elements of quantum physics, coherence and entanglement, has sparked renewed interest in the control of open quantum systems. Successful implementations face the challenge to preserve the relevant nonclassical features at the level of device operation. A major obstacle is decoherence which is caused by interaction with the environment. Optimal control theory is a tool that can be used to identify control strategies in the presence of decoherence. We review here recent advances in optimal control methodology that allow for tackling typical tasks in device operation for open quantum systems and discuss examples of relaxation-optimized dynamics. Optimal control theory is also a useful tool to exploit the environment for control. We discuss examples and point out possible future extensions.
\end{abstract}

\section{INTRODUCTION}

Control refers to the ability to steer a dynamical system from an initial to a final state with a desired accuracy; optimal control does so with minimum expenditure of effort and resources. A famous example is the Apollo space mission where optimal control was used to safely land the spacecraft on the moon. The essence of this control task can be stripped down to a textbook example where students calculate the change in acceleration, that is, the rate of burning fuel, required to reach the moon's surface with zero velocity. This example highlights the central role of optimal control in any type of engineering, its importance being rivaled only by feedback, a subject not covered in this review.

In quantum optimal control [1], Newton's equations governing the motion of the spacecraft are replaced by the quantum mechanical laws of motion, of course. In contrast, the control, corresponding for example to a radio-frequency $(\mathrm{RF})$ amplitude or the electric field of a laser, is assumed to be classical. Quantum optimal control represents one variant of quantum control [2] and is closely related to coherent control [3]. The latter requires exploitation of quantum coherence, i.e., matter wave interference. In contrast, quantum control could also refer to inducing a desired dynamics, for example by amplitude modulations that avoid driving certain transitions, without matter wave interference.

Despite of its prominence in mathematics and engineering $[4,5]$, optimal control was introduced to NMR spectroscopy $[6,7]$ and to the realm of matter wave dynamics [8-10] only in the 1980s. In the latter case, the idea was to calculate, via numerical optimization, laser fields that would steer a photoinduced chemical reaction in the desired way [8-13]. It was triggered by the advent of femtosecond lasers and pulse shaping capabilities that opened up seemingly endless possibilities to create intricate laser pulse trains. While a controlled breaking of

*Electronic address: christiane.koch@uni-kassel.de chemical bonds was indeed demonstrated soon after [14], the pulses were obtained by closed-loop optimizations in the experiments [15] rather than from theoretical calculations. In experimental closed-loop optimization, a shaped pulse is applied to the sample, and the outcome is measured. Based on the outcome, the pulse shape is modified, typically by a genetic algorithm. However, even for a chemical reaction as simple as breaking the bond in a diatomic alkali molecule, the calculated optimized laser field cannot directly be used in the experiment [16]. The reason for this is two-fold: The way how the optimized laser fields are obtained is rather different in theory and experiment. Whereas the field in calculations is shaped as a function of time $[13,17]$, experiments employ spectral shaping [18]. As a result, calculated pulses are often incompatible with experimental pulse shaping capabilities. Second, the theoretical modeling is simply not accurate enough. This results in pulses which are optimal for the 'wrong' dynamics and which can therefore not directly be applied in the experiments.

These obstacles are not present, or at least much less severe, in other fields of application [1]. Once the timescale of the relevant dynamics is nanoseconds or slower, pulse shaping in the experiment is also done in time domain [19]. While device response might still be an issue $[20,21]$, the overall approaches in theory and experiment are similar in spirit. Moreover, Hamiltonians and relaxation parameters may be known much more accurately than is currently the case in photoinduced chemical reactions. A prominent example is NMR where the development of optimal control in theory and experiment went hand in hand, yielding beautiful results, for example on arbitrary excitation profiles [22], or robust broadband excitation [23, 24].

Given these observations, quantum information processing (QIP) and related technologies offer themselves as an obvious playground for quantum optimal control: In these applications, typically the quantum system to be controlled is well characterized, and timescales are sufficiently slow to use electronics for pulse shaping. Not surprisingly, quantum optimal control has attracted much interest in these fields over the last decade. This included 
the adaptation of optimal control tools, for example to gate optimization [25-27], creation of entanglement [2830], or measurement [31]. Gate optimizations were carried out for almost all QIP platforms, notably comprising ions [32], atoms [33-35], nitrogen vacancy (NV) centers in diamond [36, 37], and superconducting qubits [38, 39]. Other QIP tasks, such as state preparation [40-44], transport [45-47], and storage [48], have also been the subject of optimal control studies. These tasks are not only relevant for quantum computing and communication but also for related applications that exploit coherence and entanglement, for example quantum sensing or quantum simulation. Protocols derived with optimal control have by now reached a maturity that allows them to be tested in experiments. Examples include the crossing of a phase transition studied with trapped, cold atoms [49, 50]; the improvement of the imaging capabilities of a single NV center [51]; and the creation of spin entanglement [52], quantum error correction [37] and matter wave interferometry $[50,53]$.

All of these examples share a generic feature that is typical for quantum engineering: Control over the system, which inevitably also brings about noise, needs to be balanced with sufficient isolation of the desired quantum features. This sets the theme for controlling open quantum systems. Traditionally, a quantum system is defined to be open when it interacts with its environment $[54,55]$. This interaction results in loss of energy and phase information. It can be modeled phenomenologically within the semigroup approach or microscopically, by embedding the system in a bath. Besides coupling to a bath, the dynamics of a quantum system becomes effectively dissipative also when the system is subject to measurements or noisy controls.

Dissipative processes pose a challenge to quantum control. At the same time, desired dissipation may act as an enabler for control. We will review control strategies in both cases and then explain how optimal control theory can be used to adapt them to more complex quantum systems.

This topical review is organized as follows: Section II briefly recalls the basic concepts in the theory of open quantum systems, introducing the distinction between Markovian and non-Markovian dynamics in Sec. II A and addressing the issue of gauging success of control for open quantum systems in Sec. II B. The problem of analyzing controllability of open quantum systems, an important prerequisiste to synthesizing control fields, is introduced in Sec. III A. Progress in the control of open quantum systems is reviewed in Sections III B and IV with Sec. III B dedicated to control strategies that were constructed with analytical methods and Sec. IV covering numerical optimal control. In Sec. IV A, the numerical methodology is explained in detail for a simple example, followed by a discussion of the modifications required to adapt it to more advanced control targets. The remainder of Sec. IV reviews applications of numerical optimal control to open quantum systems, starting with examples for fighting or avoiding decoherence in Sec. IV B. Control strategies that rely on the presence of the environment are discussed in Secs. IV C and IV D. Section V concludes.

\section{OPEN QUANTUM SYSTEMS}

The state of an open quantum system is described by the density operator $\hat{\rho}_{S}$ which is an element of Liouville space. Any theory that aims at the control of an open quantum system is faced with two basic prerequisites the ability to calculate the system's dynamics, $\hat{\boldsymbol{\rho}}_{S}(t)$, and the ability to quantify success of control.

\section{A. Markovian vs non-Markovian dynamics}

Formally, the time evolution of any open quantum system can be described by a dynamical map, $\hat{\boldsymbol{\rho}}_{S}(t)=$ $\mathcal{D}_{t, 0}\left(\hat{\boldsymbol{\rho}}_{S}(0)\right)$ which is completely positive and trace preserving (CPTP) [55]. The dynamical map is divisible if it can be written as the composition of two CPTP maps $\mathcal{D}_{t, 0}=\mathcal{D}_{t, t^{\prime}} \mathcal{D}_{t^{\prime}, 0} \forall t^{\prime} \leq t$. If the dynamical map is divisible, the open system's time evolution is memoryless and called Markovian. Various scenarios can lead to Markovian dynamics, weak coupling between system and environment together with a decay of environmental correlations much faster than the timescales of the system dynamics being the most common case [55]. However, open systems often exhibit pronounced memory effects, in particular in condensed matter experiments, which reflect characteristic features of the environment. The dynamics are then called non-Markovian. Memory effects are caused by structured spectral densities, nonlocal correlations between environmental degrees of freedom and correlations in the initial system-environment state $[56,57]$.

In the Markovian case, the dynamics can be described by a master equation in Lindblad form [55]. In general, it needs to be solved numerically to determine $\hat{\boldsymbol{\rho}}(t)$. This can be done with arbitrarily high precision [58]. However, the computational effort may quickly become challenging due to the exponential scaling of the size of Hilbert and Liouville space. To date, room for improvement seems to be limited [59,60].

The situation is worse for non-Markovian dynamics, where a unified framework such as the master equation in Lindblad form does not exist. A variety of methods has been developed [61], each with different assumptions and hence a different range of applicability. They include time-local non-Markovian master equations [55], stochastic unravellings [62-64], and an auxiliary density matrix approach [65]. A common feature of these methods is their ability to correctly describe thermalization of the system. Slightly different in philosophy are methods which attempt to solve the dynamics of both system and environment [66, 67]. Key is to account only for that part of the environment that is relevant for the system's dynamics, i.e., for the 'effective modes', which can be spins 
or harmonic oscillators. The underlying idea is that of quantum simulation on a classical computer [68], where the true environment is replaced by a surrogate one that generates the same dynamics. If one is interested in short times, the number of modes in the surrogate Hamiltonian can be truncated with a prespecified error [69]. Longer propagation times than those computationally affordable with exact dynamics of system and environment become possible by separating the environment into two baths, one that is responsible for the memory effects and that is modeled by effective modes as explained above, and a second one that by itself would lead to Markovian dynamics only. The secondary bath can be accounted for in terms of a Markovian master equation in Lindblad form [38, 70] or via a stochastic unravelling using a single secondary bath mode [71]. A more comprehensive overview over methods to tackle non-Markovian dynamics is found in Ref. [61].

Understanding the influence of memory effects requires the ability to quantify them. An obvious way to define a measure of non-Markovianity is to quantify deviation from divisibility $[72,73]$. Interestingly, this corresponds to an increase of correlations if the system is bi- or multipartite $[73,74]$. Other measures to capture memory effects focus on specific features such as recoherence and information backflow. These can be characterized in terms of distinguishability between quantum states [75-77], reexpansion of the volume of accessible states [78], or the capacity to reliably transmit quantum information [79]. A comprehensive overview over the different measures is found in Ref. [56], and an illustrative comparison for a toy model is presented in Ref. [80].

The proposed non-Markovianity measures can be classified in a hierarchy by generalizing the notion of divisibility [81, 82]. While this is gratifying from a theoretical perspective, it is still an open question how nonMarkovianity can be measured in a condensed phase experiment. Although some of the measures have been evaluated in experiment [83, 84], dissipation in these examples was either engineered or artificial, in the sense that different degrees of freedom within one particle were considered to play the role of system and bath. A true condensed phase setting is more challenging due to limited control and thus limited access to measurable quantities.

Current interest in non-Markovian dynamics is fueled by the revival of genuine quantum properties such as quantum coherence and correlations that nonMarkovianity entails. It has sparked the hope to exploit non-Markovianity as a resource. Quantum control in particular, which relies on properties such as coherence, should be more powerful in the non-Markovian compared to the Markovian regime.

\section{B. Measuring success of control in open quantum systems}

When the goal is to control an open quantum system, the ability to gauge success of control is even more important than that to measure the degree of nonMarkovianity. Any suitable figure of merit needs to fulfill two conditions: (i) It should take its optimum value if and only if the control target is reached. (ii) It needs to be computable. An obvious control target is to drive a given initial state to a desired target state. The corresponding figure of merit is the projection onto the target state. For open quantum systems, this is given in terms of the Hilbert Schmidt product of the state of the system at the final time and the target state,

$$
F_{T}=\operatorname{Tr}\left\{\mathcal{D}_{T, 0}\left(\hat{\boldsymbol{\rho}}_{\text {initial }}\right) \hat{\boldsymbol{\rho}}_{\text {target }}\right\}
$$

This figure of merit has been used for example in control studies of cooling where the timescales of the dissipative process and the coherent system dynamics are comparable $[85,86]$. Typically, the target for cooling is a pure state. Sometimes the timescale of dissipation is much slower than that of the coherent dynamics. This situation is encountered when using femtosecond lasers for laser cooling [87]. In order to avoid the very long propagation times for repeated cooling cycles consisting of coherent excitation and spontaneous emission as well as the expensive description in Liouville space, one can expand $\hat{\boldsymbol{\rho}}_{\text {initial }}$ in a basis of Hilbert space vectors and tailor the dynamics of the Hilbert space vectors such that the target will be reached irrespective of the initial state [88]. The construction of the proper figure of merit in that case will be discussed below in Section IV C.

An important control target in the context of quantum information processing is the implementation of unitary operations, or quantum gates. This corresponds to simultaneous state-to-state transitions for all states in the logical basis [25-27]. A straightforward way to express this control target in Liouville space is given by $[89,90]$

$$
F_{T}=\frac{1}{d} \sum_{j=1}^{d^{2}} \operatorname{Tr}\left\{\hat{\mathbf{0}} \hat{\boldsymbol{\rho}}_{j} \hat{\mathbf{O}}^{+} \mathcal{D}_{T, 0}\left(\hat{\boldsymbol{\rho}}_{j}\right)\right\},
$$

where $\mathbf{O}$ denotes the desired target operation, defined on the logical subspace of dimension $d$. The set of $\hat{\boldsymbol{\rho}}_{j}$ forms a suitable orthonormal basis of the $d^{2}$-dimensional Liouville (sub)space or, more simply, all $d^{2}$ matrices for which one entry is equal to one and all other zero. The Hilbert Schmidt product in Eq. (2) checks how well the actual evolution, $\mathcal{D}_{T, 0}\left(\hat{\boldsymbol{\rho}}_{j}\right)$, matches the desired one, $\mathbf{\mathbf { O }} \hat{\boldsymbol{\rho}}_{j} \hat{\mathbf{O}}^{+}$. The scaling of Eq. (2) with system size $d$ restricts its use to examples with very few qubits.

If the target is the implementation of a unitary operation, and not an arbitrary dynamical map, much less resources are required to gauge success of control. This observation is at the basis of all current proposals to estimate the average gate fidelity [91-95] which forego the 
full knowledge of $\mathcal{D}_{T, 0}$ that is obtained in quantum process tomography in favor of efficiency. One way to understand the reduction in effort is to start from Eq. (2) and ask how many states are required in the sum to have a well-defined figure of merit, i.e., a figure of merit that takes its optimum value if and only if the target operation is realized. Surprisingly, the answer to this question is three, independent of system size [39, 96]:

$$
F_{T}=\frac{1}{3} \sum_{j=1}^{3} \operatorname{Tr}\left\{\hat{\mathbf{O}} \hat{\boldsymbol{\rho}}_{j} \hat{\mathbf{O}}^{+} \mathcal{D}_{T, 0}\left(\hat{\boldsymbol{\rho}}_{j}\right)\right\} .
$$

One state in Eq. (3) measures the departure from unitarity or, more precisely, from unitality in the logical subspace, and two more states are necessary to distinguish any two unitaries [211]. The latter requires two states because one needs to determine the basis in which the unitary is diagonal and then check whether the phases on the diagonal are identical. Only two states are required because it is possible to construct one density operator that 'fixes' the complete Hilbert space basis, $\hat{\boldsymbol{\rho}}_{2}=\sum_{i=1}^{d} \lambda_{i}\left|\varphi_{i}\right\rangle\left\langle\varphi_{i}\right|$, using one-dimensional orthogonal projectors, $\left|\varphi_{i}\right\rangle\left\langle\varphi_{i}\right|$, with non-degenerate eigenvalues, $\lambda_{i} \neq \lambda_{j}$. A variant of Eq. (3) is obtained by replacing the two states for the basis and phases by $d$ states $\rho_{j}=\left|\varphi_{i}\right\rangle\left\langle\varphi_{i}\right|$. This is still a reduction compared to the $d^{2}$ states in Eq. (2) and was found to lead to faster convergence in control calculations than Eq. (3) [39]. Evaluation of both Eq. (3) and its $d+1$ state variant are much more efficient than that of Eq. (2) [39].

Both Eqs. (2) and (3) target implementation of a specific unitary Ô. For difficult control problems, where a numerical search can easily get stuck, it is desirable to formulate the control target in the most flexible way. For example, instead of implementing a specific two-qubit gate such as CNOT, it may be sufficient to realize a gate that is locally equivalent to CNOT, i.e., that differs from CNOT by local operations. The corresponding figure of merit is based on the so-called local invariants [29, 97]. Similarly, one can formulate a figure of merit for targeting an arbitrary perfect entangler [98, 99]. Since these figures of merit are based on the local invariants which in turn are calculated from the unitary evolution, extension to non-unitary dynamics requires to first determine the unitary part of the overall evolution. This is possible, using the same mathematical concepts that have led to Eq. (3) [100].

\section{CONTROL OF OPEN QUANTUM SYSTEMS}

The theory of controlling open quantum system can be divided into two main questions - analysis of controllability and synthesis of controls (called motion planning in the classical automatic control community): When the goal is to realize a certain desired dynamics, it is worthwhile to check first whether performance of the task is possible at all, before starting to search for controls that lead to the target. Section III A briefly reviews the current state of the art in controllability analysis of open quantum systems, whereas Sec. III B summarizes strategies for control synthesis that are based on certain properties of the system's interaction with its environment, and optimal control theory as a tool for control synthesis will be presented in Sec. IV.

\section{A. Controllability of open quantum systems}

Controllability analysis provides the mathematical tools for answering the question whether the target is reachable [101]. In particular, a complete framework exists for finite-dimensional systems undergoing unitary dynamics: Separating the Hamiltonian into drift and control terms,

$$
\hat{\mathbf{H}}=\hat{\mathbf{H}}_{0}+\sum_{j} u_{j}(t) \hat{\mathbf{H}}_{j}
$$

the system is controllable provided the Lie algebra spanned by the nested commutators of $\hat{\mathbf{H}}_{0}$ and $\hat{\mathbf{H}}_{j}$ is full rank $[2,102,103]$. The elements of the Lie algrebra can be interpreted as tangential vectors, i.e., as the directions, of the unitary evolution (which is an element of a Lie group). If evolution into all directions can be generated, the system is controllable. Controllability can also be viewed in terms of connectivity between Hilbert space basis states [104]; it then corresponds to presence of the respective matrix elements.

For open quantum systems, the evolution is not unitary anymore, and analysis of the Hamiltonian alone is not sufficient to decide controllability: The dissipative part of the evolution may prevent or enable certain states to be reached. For example, even if the full rank condition for the Lie algebra of the Hamiltonian is fulfilled, fast decoherence will inhibit transitions between pure states by turning any pure state into a mixed one. Merely the presence of non-vanishing matrix elements in the Hamiltonian is thus not sufficient to decide controllability. Their magnitude matters as well, in particular of those in the drift Hamiltonian $\hat{\mathbf{H}}_{0}$ that cannot be tuned by external controls $u_{j}$. To date, rigorous controllability analysis does not take such a dependence on operator norms, or competing time scales into account.

As a result, one needs to turn to numerical search for most open quantum systems, even for an example as simple as the central spin model [105]. This is rather unsatisfactory since a numerical search cannot provide rigorous answers to controllability, in particular lack thereof (in the sense of reachability of the target with a predetermined error $\epsilon$ ), due to its local character. An extension of controllability analysis to the needs of open quantum systems would address this issue but remains an open problem to date. It would be particularly relevant for open quantum systems with almost unitary dynamics, 
which are often encountered e.g. in quantum technology applications.

As an example where the dissipative part of the overall evolution enables certain states to be reached, consider cooling which turns mixed states into pure ones. Obviously, controllability analysis based on the Lie rank condition for the Hamiltonian alone cannot provide any information on time evolutions which change the purity of the system's state, $\mathcal{P}=\operatorname{Tr}\left[\hat{\boldsymbol{\rho}}_{S}^{2}(t)\right]$.

By and large, controllability of open quantum systems still remains uncharted territory to date [1]. This refers in particular to dynamic controllability where the analysis accounts for available dynamical resources such as coupling to external fields and environmental degrees of freedom, or measurements, in contrast to kinematic controllability. The latter implies existence of a dynamical map that transforms any initial state into the target state. While this existence can be proven for finitedimensional systems [106], it is of limited relevance for practical applications since, in general, one cannot derive any dynamical information from the proof.

To tackle controllability of open quantum systems, two routes can be followed: Either one starts from a complete description of the system and its environment, or one considers the reduced description of the system alone. In the first case, the tools of controllability analysis for unitary dynamics can be employed. This way it was possible to show, for example, that even for completely controllable system-environment dynamics, cooling is possible only if the environment contains a sufficiently large virtual subsystem which is in a state with the desired purity [107]. While exact solution of the combined systemenvironment dynamics is extremely challenging (and impossible in many cases), controllability analysis from this perspective is expected to significantly advance our understanding already for simple models and for generic questions, as in the example of Ref. [107]. Moreover, it appears to be promising for two reasons - it does not rely on a priori assumptions on the reduced dynamics, and most likely it will benefit from recent progress in the controllability of infinite-dimensional systems [108-111].

Rigorous controllability analyses for reduced dynamics have been limited to date to the Markovian case [112117]. In particular, the sets of reachable states were characterized [112], and the Lindblad operators were shown to form a Lie wedge [114]. While the Lie wedge provides a sufficient, but not necessary condition for controllability, necessary but not sufficient criteria can be identified by considering isotropy of the generator of the dissipative motion [117]. Numerically, non-Markovian dynamics were shown to lead to $S U(N)$ controllability (in the sense of reachability of the target with a predetermined error $\epsilon$ ) for a system whose Hamiltonian allows for realization of $S O(N)$ operations only [70]. However, no rigorous analysis of controllability for non-Markovian dynamics has been performed to date; and it is not yet clear whether and under which conditions non-Markovian effects can improve controllability of an open quantum system.

\section{B. Control strategies for open quantum systems}

Control strategies that are obtained by analytical methods can be roughly divided into two classes - those that exploit symmetries in the system-bath interaction and those that make assumptions on the timescale of this interaction. In the first case, protection from decoherence is achieved by keeping the system's state in a so-called decoherence-free subspace [118, 119]: If the system-bath interaction contains a symmetry, for example qubits couple indistinguishably to their environment, it is possible to construct a set of system states that are invariant under the system-bath interaction. These states form a decoherence-free subspace in the system's total Hilbert space [118, 119]. Stimulated Raman Adiabatic Passage (STIRAP) and electromagnetically induced transparency represent examples for decoherence-free subspaces [119]. In the context of quantum information, two or more physical qubits, carried for example by atoms, can be used to encode one logical qubit that is decoherence-free [120]. Decoherence-free subspaces have been demonstrated, for example, in liquid-state nuclear magnetic resonance [121] and with trapped ions [122]. Numerical methods can be employed to identify (approximate) decoherence-free subspaces [123] and to find an external control that drives the system dynamics into a decoherence-free subspace [124].

More generally, in physics the presence of a symmetry implies existence of a conserved quantity. In the context of decoherence, a symmetry in the system-bath interaction leads to a quantum number which is preserved under this interaction. The eigenstates belonging to the preserved quantum number define a noiseless subsystem, i.e., a logical subsystem that is intrinsically protected from noise $[125,126]$. The main limitation of this set of approaches is imposed by the existence of a suitable symmetry which is not necessarily available.

In the second case of control strategies that are built on assumptions on the timescale of the system-bath interaction, a trivial strategy is obtained for slow decoherence: One simply needs to perform the desired operation on a time scale much faster than that of decoherence. But this may not always be possible, and slow decoherence also allows for eliminating the effects of decoherence based on average Hamiltonian theory [127] or, in more intuitive terms, spin echo techniques from nuclear magnetic resonance and generalizations thereof. This set of strategies is often referred to as dynamical decoupling [128-131]. It relies on many quasi-instantaneous actions of control fields that do not allow the system to interact with its environment, creating an effective dynamics of the system alone.

Extensive work over the last two decades has allowed to account for e.g. pulse imperfections [132] and extend the technique beyond spin dynamics for which it was originally developed, for example to ions [133], and superconducting circuits [134]. The main limitation of the dynamical decoupling approach is the finite duration of any control field which cannot always be made suffi- 
ciently short. By now dynamical decoupling has grown into a field of its own which has been covered by several reviews [129-131], and the reader is referred to these for a more in-depth analysis.

The time-dependent perspective used in dynamical decoupling can be translated into a frequency-space picture using the generalized transfer filter function approach $[135,136]$. This allows to efficiently predict fidelities, at least for weak noise [137], and provides a connection to control strategies that decouple the system from its environment by engineering a spectral separation [138]. In particular, it was shown that the systembath interaction can be cancelled, at least to second order, by choosing the time-dependence of the control field such that its spectrum becomes orthogonal to the bath or noise spectrum [138]. Such an approach requires weak coupling and negligible initial system-bath correlations. Moreover, the bath spectrum needs to be known. Another example of engineering a spectral separation is given by the protection of so-called edge states in topological insulators via band Liouvillians [139, 140].

In addition to clarifying the relation between timedomain based and frequency-domain based control strategies, the transfer filter function perspective also provides a practical tool for characterizing the noise spectral density [141]. Its applicability to a variety of physical platforms has already been demonstrated [141143]. Noise spectroscopy provides an excellent starting point for deriving microscopic models for the systemenvironment interaction and thus a more thorough understanding of noise at the quantum mechanical level. It also allows for tailoring control synthesis to specific spectral features of the noise, either, if possible, by using the transfer filter function approach directly [136], or by exploiting knowledge of these features using optimal control theory, in Sec. IV below.

\section{OPTIMAL CONTROL OF OPEN QUANTUM SYSTEMS}

Quantum optimal control theory refers to a set of methods that synthesize external control fields from knowledge of the control target, including constraints, and the time evolution of the quantum system [1]. It is based on the calculus of variations, i.e., on formulating the control target as a functional of external controls that realize the desired dynamics. Knowledge of the system's time evolution enters implicitly via evaluation of the target functional and, possibly, its derivatives.

For a few exceptional cases, for example one or two spins (or qubits) [144-149], a harmonic oscillator $[150,151]$, or a sequence of $\Lambda$-systems subject to decay $[152,153]$, the external controls can be determined using geometric techniques based on Pontryagin's maximum principle [4]. Typically, however, the control problem cannot be solved in closed form, and one needs to resort to numerical optimization.
Most often, controlling open quantum systems is a difficult control task such that efficiency of the optimization algorithm is important. Therefore, mainly algorithms based on the target functional's gradient have been employed for open quantum systems to date. They will be reviewed in Sec. IV A. The remainder of this section is dedicated to control strategies that were developed using numerical optimization, starting with strategies avoiding decoherence in Sec. IV B, followed by strategies exploiting presence of the environment in Sec. IV C and IV D.

\section{A. Optimal control theory applied to open quantum systems}

Conceptually the simplest control problem is represented by state-to-state transfer: Given a known initial state, $\hat{\boldsymbol{\rho}}_{\text {ini }}$, at time $t=0$, find the external field that drives this state at final time $t=T$ into the target state, $\hat{\boldsymbol{\rho}}_{\text {target }}$, with prespecified error, $\epsilon$. The corresponding target functional is found in Eq. (1) where dependence on the set of external controls $\left\{u_{j}\right\}$ is implicit in the time evolution, $\mathcal{D}_{T, 0}=\mathcal{D}_{T, 0}\left[\left\{u_{j}\right\}\right]$.

This control problem was first stated in the context of laser cooling molecular vibrations $[85,154,155]$. The time evolution was modeled by a Markovian master equation in Lindblad form, and later extended to a nonMarkovian example $[86,156]$. For the sake of conceptual clarity, we present here the algorithm for a Markovian master equation,

$$
\frac{d \hat{\boldsymbol{\rho}}}{d t}=\mathcal{L}(\hat{\boldsymbol{\rho}})=-i[\hat{\mathbf{H}}[u(t)], \hat{\boldsymbol{\rho}}]+\mathcal{L}_{D}(\hat{\boldsymbol{\rho}}),
$$

with the Hamiltonian of the form (4). For simplicity, we assume a single control $u_{1}(t)=u(t)$ in Eq. (4). In the example of laser cooling molecular vibrations, $u(t)$ would be the electric field of a short laser pulse, and $\hat{\mathbf{H}}_{1}$ in Eq. (4) the transition dipole moment of the molecule. The dissipative part of Eq. (5) is given by [55]

$$
\mathcal{L}_{D}(\hat{\boldsymbol{\rho}})=\sum_{k} \gamma_{k}\left(\hat{\mathbf{A}}_{k} \hat{\boldsymbol{\rho}} \hat{\mathbf{A}}_{k}^{+}-\frac{1}{2}\left\{\hat{\mathbf{A}}_{k}^{+} \hat{\mathbf{A}}_{k}, \hat{\boldsymbol{\rho}}\right\}\right),
$$

where the Lindblad operators $\hat{\mathbf{A}}_{k}$ model the various dissipative channels. For example, $\hat{\mathbf{A}}_{k}=|0\rangle\langle k|$ for spontaneous decay from a level $k$ to the ground state with rate $\gamma_{k}$, inversely proportional to the level's lifetime.

Optimization algorithms are obtained by seeking an extremum of $F_{T}$, cf. Eq. (1), with respect to the control, $u(t)$. This can be done by direct evaluation of the extremum condition $[157,158]$ or by building in monotonic convergence a priori using Krotov's method [85, 97]. The resulting set of coupled equations are, surprisingly, rather similar. The main difference is in the update of the control which can be performed concurrently [158], i.e., for all times $t$ at once, or sequentially in time [85, 97, 157]. Guaranteed monotonic convergence is only obtained with 
a sequential update of the control. In this case, the equation for determining the control reads

$$
\begin{aligned}
\Delta u(t) & =u^{(i+1)}(t)-u^{(i)}(t) \\
& =\frac{S(t)}{\lambda} \mathfrak{I m}\left\{\operatorname{Tr}\left[\left.\hat{\boldsymbol{\sigma}}^{(i)}(t) \frac{\partial \mathcal{L}(\hat{\boldsymbol{\rho}})}{\partial u}\right|_{\rho^{(i+1)}, u^{(i+1)}}\right]\right\} .
\end{aligned}
$$

$S(t)$ denotes a shape function that can be used to switch the control on and off smoothly [159] or to impose an initial or final ramp [70]; and $\lambda$ is a parameter of the algorithm whose choice determines the step size in the change of the control. Its optimal value can be estimated in an automated way, similarly to a line search in quasiNewton methods [160]. $\quad \hat{\boldsymbol{\sigma}}(t)$ denotes the so-called costate or adjoint state, and the derivative with respect to the control is given by the commutator

$$
\left.\frac{\partial \mathcal{L}(\hat{\boldsymbol{\rho}})}{\partial u}\right|_{\rho^{(i+1)}, u^{(i+1)}}=-i\left[\left.\frac{\partial \hat{\mathbf{H}}[u(t)]}{\partial u}\right|_{u^{(i+1)}}, \hat{\boldsymbol{\rho}}^{(i+1)}\right] .
$$

For the common case of linear coupling to the control, as in Eq. (4), the explicit dependence on $u^{(i+1)}$ vanishes and the commutator simply becomes $\left[\hat{\mathbf{H}}_{1}, \hat{\boldsymbol{\rho}}^{(i+1)}\right]$. Nonetheless, the right-hand side of Eq. (7) depends on $u^{(i+1)}$ via $\hat{\boldsymbol{\rho}}^{(i+1)}(t)$, i.e., it is an implicit equation. Solution of the implicit equation can usually be avoided by a low order approximation in the iterative algorithm, employing two shifted time discretizations to represent the time dependence of states and control, $\hat{\boldsymbol{\rho}}\left(t_{i}\right)$ and $u\left(t_{i}+\Delta t / 2\right)$ [27].

Equation (7) also depends on the state of system at time $t, \hat{\boldsymbol{\rho}}(t)$, and the co-state, $\hat{\boldsymbol{\sigma}}(t)$. These are obtained by solving

$$
\frac{d \hat{\boldsymbol{\rho}}^{(i)}}{d t}=-i\left[\hat{\mathbf{H}}\left[u^{(i)}(t)\right], \hat{\boldsymbol{\rho}}^{(i)}\right]+\mathcal{L}_{D}\left(\hat{\boldsymbol{\rho}}^{(i)}\right)
$$

with initial condition

$$
\hat{\boldsymbol{\rho}}^{(i)}(t=0)=\hat{\boldsymbol{\rho}}_{\text {ini }},
$$

and

$$
\frac{d \hat{\boldsymbol{\sigma}}^{(i)}}{d t}=-i\left[\hat{\mathbf{H}}\left[u^{(i)}(t)\right], \hat{\boldsymbol{\sigma}}^{(i)}\right]-\mathcal{L}_{D}\left(\hat{\boldsymbol{\sigma}}^{(i)}\right),
$$

which is solved backward in time. The 'initial' condition is derived from the target functional at final time $F_{T}$,

$$
\hat{\boldsymbol{\sigma}}^{(i)}(t=T)=\hat{\boldsymbol{\rho}}_{\text {target }}^{(i)} .
$$

This coupled set of equations needs to be solved iteratively, starting with some guess for the control, $u^{(i=0)}(t)$, where the index $(i)$ denotes iteration.

The algorithm represented by Eqs.(7), (8) and (9) is straightforwardly extended from targeting a single state to targeting a unitary operation $[39,89,90]$ : A unitary operation $\hat{\mathbf{U}}$ can be viewed as several simultaneous stateto-state transfers $[26,27]$ which are all driven by the same control. Consequently, Eq. (7) becomes

$\Delta u(t)=\frac{S(t)}{\lambda} \sum_{j=1}^{M} \mathfrak{I m}\left\{\operatorname{Tr}\left[\left.\hat{\boldsymbol{\sigma}}_{j}^{(i)}(t) \frac{\partial \mathcal{L}\left(\hat{\boldsymbol{\rho}}_{j}\right)}{\partial u}\right|_{\rho_{j}^{(i+1)}, u^{(i+1)}}\right]\right\}$,

and Eqs. (8) and (9) need to be solved for $M$ states $\hat{\boldsymbol{\rho}}_{j}(t)$ and $M$ co-states $\hat{\boldsymbol{\sigma}}_{j}^{(i)}(t)$ simultaneously. As explained in Sec. II B, it was first believed that the sum in Eq. (10) has to run over $M=d^{2}$ states where $d$ is the dimension of the space on which the desired operation is defined [89, 90]. The initial conditions $\hat{\boldsymbol{\rho}}_{j}(t=0)$ are then given by orthogonal basis states spanning this space. Recently it was shown that $M$ can be reduced all the way down to 3 in which case the states discussed in Sec. II B need to be taken as initial conditions $\hat{\boldsymbol{\rho}}_{j}(t=0)$ [39]. The initial conditions for the co-states are always given in terms of the desired target operation, $\hat{\boldsymbol{\sigma}}_{j}(t=T) \sim \hat{\mathbf{U}} \hat{\boldsymbol{\rho}}_{j}(t=0) \hat{\mathbf{U}}^{+}$, up to a suitable normalization [39]. Moreover, weights may be introduced in $\hat{\boldsymbol{\sigma}}_{j}(t=T)$ to speed up convergence by attaching different importance to different basis states $\hat{\boldsymbol{\rho}}_{j}(t=0)[39]$.

Extension of the optimization algorithm to more flexible control targets, such as an arbitrary perfect entangler [98, 99] instead of a specific unitary, requires two steps. First, a modified target functional results in a modification of Eq. (9b): The right-hand side of Eq. (9b) will be replaced by the derivative of the new target functional with respect to the states, $\hat{\boldsymbol{\rho}}_{j}$, evaluated at time $t=T$. For a target functional based on the local invariants, this requires, in particular, to determine the unitary part of the overall actual evolution, as explained in Sec. II B. Second, dependence of the functional on the states $\hat{\boldsymbol{\rho}}_{j}$ may be non-convex. In this case, Eq. (10) needs to be amended by a second term in its right-hand side [97] which depends additionally on the change in the states, $\hat{\boldsymbol{\rho}}_{j}^{(i+1)}-\hat{\boldsymbol{\rho}}_{j}^{(i)}$. While the additional computational effort for evaluating the control update is negligible, storage of all $\hat{\boldsymbol{\rho}}_{j}^{(i)}$ is necessary and may become a limiting factor when scaling up the system size. Such extensions of the optimization algorithm to control targets other than a specific state or unitary have been applied to coherent dynamics [29, 97-99]. For open quantum systems, they are still under exploration.

Typically, more than one solution exists to a quantum control problem. When using the basic optimization algorithm presented above, it will then depend on the initial guess $u^{(0)}(t)$ which solution $u^{\star}(t)$ the algorithm identifies. Two strategies can be employed in order to fine-tune the iterative search and guide it toward a solution with certain desired properties - careful selection of the initial guess by scanning or preoptimization [161] or use of additional constraints $[162,163]$. In the first case, the initial guess needs to be parametrized in a suitable form, for example in terms of amplitudes and phases of Fourier components, or amplitudes, widths and positions of Gaussians. These parameters can easily be preoptimized within a prespecified range employing a stan- 
dard gradient-free optimization method [161]. When the result is used as initial guess in the optimization algorithm presented above, the ensuing fine-tuning will usually not lead to drastic changes in the field, keeping it close to the parametrized form [161].

A more stringent way to enforce certain desired properties of the control solution is obtained by employing additional constraints $[162,163]$. This comes at the expense of a modified optimization algorithm. An explicit description for deriving the modified algorithms is available when using Krotov's method [97]. It allows for formulating constraints as a functional of the control, with the only condition that the functional be positive (or negative) semidefinite [163]. This requirement is necessary to ensure monotonic convergence. As an example, consider a spectral constraint on the control [163],

$$
J_{\text {spec }}[u(t)]=\frac{1}{2 \pi} \int_{0}^{T} \int_{0}^{T} \Delta u(t) K\left(t-t^{\prime}\right) \Delta u\left(t^{\prime}\right) d t^{\prime} d t
$$

where $K\left(t-t^{\prime}\right)$ is the Fourier transform of a spectral filter $\tilde{K}(\omega) \geq 0$. As a result, the update equation for the control becomes a Fredholm equation of the second kind [163]. A judicious choice of the shape function $S(t)$ allows for analytically solving the Fredholm equation in frequency domain such that the additional numerical effort for including the spectral constraint consists merely in two additional Fourier transforms [164]. More than one constraint may be employed at a time, with different weights allowing to emphasize importance of one compared to another $[162,164]$. While additional constraints provide information that guides the optimization algorithm, they also restrict the space of admissible solutions $[165,166]$. Therefore care needs to be taken to balance their benefits and their disadvantages.

\section{B. Fighting and avoiding decoherence}

While it is probably the dream of every control engineer to discover unthought of control schemes, a more realistic scenario starts from known a control strategy, as those described in Sec. III B, and extends it to a wider range of conditions, new types of systems, or new types of dissipative processes using the optimization techniques described above.

One of the most popular control strategy in the area of quantum technologies currently is dynamical decoupling $[130,131]$. While already powerful in itself, dynamical decoupling can be made more robust by numerical optimization that targets specific noise features that were previously unaccounted for, using, for example, the gradient-ascent technique [167] or genetic algorithms [168]. In these examples, optimization did not compromise feasibility of the pulse sequences. Moreover, the length of dynamical decoupling sequences can be minimized [169]. Dynamical decoupling and numerically optimized pulses can also complement each other, as recently demonstrated for entanglement generation and distribution in NV centers in diamonds [52].

A second successful control strategy described in Sec. IIIB is based on decoherence-free subspaces and noiseless subsystems. These are somewhat less often used than dynamical decoupling, mainly due to the difficulty of identifying them for more complex systems. While direct identification of decoherence-free subspaces is hampered by presence of numerous traps in the search space [123], quantum optimal control may be used to dynamically identify them. Indeed, optimization of an open system's dynamics for targets that rely on quantum coherence is intrinsically biased toward those subspaces in Hilbert (or Liouville) space that are least affected by decoherence [170]. For example, transfer of coherence and polarization between coupled heteronuclear spins was improved by cross-correlated relaxation optimized pulse (CROP) sequences and relaxation optimized pulse elements (ROPE) [144, 145, 171, 172]. Optimization can take both longitudinal and transveral relaxation into account [171]. The underlying mechanism was revealed to consist in tuning cross-correlated to autocorrelated relaxation rates [144]. Counterintuitively, maximum polarization transfer between coupled spins was achieved with sequences that are longer than conventional ones [145], highlighting the importance to include the dissipative dynamics in the optimization.

Finally, if all regions in Hilbert space are similarly affected by decoherence, an obvious control strategy consists in beating decoherence by the fastest possible operation. This strategy is faced, however, with the socalled quantum speed limit, i.e., a fundamental bound on the shortest operation time $[173,174]$. For a twolevel system, it can be estimated analytically [175]. For more complex systems, optimal control theory can be used as a tool to both identify the quantum speed limit and determine controls that drive the system at the quantum speed limit [46]. For example, the shortest possible duration of entangling quantum gates was determined for cold, trapped atoms [34] and for superconducting qubits $[39,176]$. In quantum dots, phonon-assisted decoherence was minimized [177, 178]. Interestingly, presence of the environment may improve the quantum speed limit $[179,180]$. This has not yet been explored systematically but could be done, using quantum optimal control.

\section{Cooling and quantum reservoir engineering}

The example of cooling $[85,86,88,148,149,155,156$, $181,182]$ was already taken as reference to introduce optimal control of open quantum systems in Sec. IV A. Since cooling changes the purity of the state, it relies on the presence of the environment. When using the algorithm outlined in Sec. IV A, the control that drives the cooling process is determined for each initial state separately. Alternatively, one may seek a control that will lead to cooling irrespective of the initial state [88]. Such 
an approach is particularly useful if the timescales of the coherent and the dissipative dynamics is different, as in the case of optical pumping [87] which consists of many repeated cycles of excitation and spontaneous emission. The theoretical framework lends itself to generalization to quantum reservoir engineering which is why it is outlined in more detail in the following.

The idea is to start from an orthogonal basis for the space on which the initial states are defined and ensure transitions favorable to cooling for every basis state [88]. In the case of optical pumping, these are transitions into states from which spontaneous emission preferrably occurs to the cooling target. As is usual in quantum optimal control, this control task is stated in terms of a yield functional. Additionally, the control should not excite any population which has already been accumulated in the cooling target state. In other words, the cooling target should remain a steady state of the evolution. This requirement is translated into a second term in the optimization functional, besides the yield. Moreover, it is often not possible to cool arbitrary initial states, due to limitations on control bandwidth. Instead, one can seek to cool states which are defined on a certain subspace. This results in an additional term in the optimization functional that suppresses leakage from this subspace in order to keep the cooling cycle closed. Finally, one needs to guarantee that for all basis states cooling occurs with the same efficiency; otherwise the cooling might get stuck. This can be achieved either by imposing symmetric excitation of all basis states or by having all basis states form an 'assembly line', i.e., enforce one specific excitation pathway for all states. All requirements need to be met simultanously and, consequently, the optimization functional consists of four terms - one for the yield, one of the steady state, one to suppress leakage and one to ensure symmetric excitation or enforce a specific excitation pathway for all states [88].

Application of this optimization framework showed that laser cooling of molecular vibrations is possible even in cases where the molecular structure favors heating rather than cooling [88]. It also answers the question about the minimal requirement on the molecular structure to realize, with shaped pulses, cooling instead of heating, assuming no constraints on the control - existence of one state which undergoes spontaneous emission with moderate probability into the cooling target.

If the molecular structure is favorable to cooling vibrations, an optimized laser pulse results in a substantially smaller number of cooling cycles than an unshaped pulse [88]. A similar speed up of the cooling due to pulse sequences obtained from quantum optimal control theory has also been reported for an optomechanical resonator [181] and for trapped, quasi-condensed cold atoms [182].

Laser cooling can be viewed as a particular example of quantum reservoir engineering [183-185], where a desired state becomes the 'ground state' of a driven dissipative system. It holds the promise of a particularly robust control strategy. However, applications of quantum reservoir engineering have been limited to quantum optics to date. In a condensed phase settings, both desired and undesired dissipative channels come into play, and non-Markovian effects may occur. Thus, quantum reservoir engineering in the condensed phase represents a challenging control problem.

As with any control problem, two questions need to be tackled - that of controllability and that of control synthesis, i.e., what states are attainable and how can the necessary driving be realized. The first question has been answered for generic models, such as a two- and a four-level system and a harmonic oscillator, that undergo Markovian dynamics [186]. The obtained understanding of controllability can be exploited to construct dissipative channels that allow for the robust generation of long-distance entanglement [187]. The question which states are attainable in the presence of additional undesired dissipative channels, a generic feature of any condensed phase setting, has not yet been tackled to date. A possible influence of non-Markovian effects on the reachable states has also not yet been addressed.

Control synthesis may be achieved in several ways. First, incoherent control by the environment, for example, via certain population distributions in the environmental modes, may be used to control the system [188]. However, this contradicts the assumption that the environment by definition is uncontrollable. Alternatively, measurements effectively lead to dissipative dynamics and may thus be used to generate desired dissipation [189-191]. They may be augmented by suitably tailored coherent excitation for more effective control [192]. Quantum reservoir engineering may also be formulated as an optimization problem where the target is a certain desired steady state. An optimization algorithm is obtained by generalizing the theoretical framework for laser cooling [88] outlined above. However, the search space is even larger than for a standard quantum control problem; and the efficient numerical implementation is an open challenge. Meeting this challenge would allow for exploring quantum reservoir engineering in the presence of undesired dissipation typical for condensed phase settings and in the non-Markovian regime.

\section{Exploiting non-Markovianity for quantum control}

It was shown already several years ago that nonMarkovian evolution may ease control [38, 193], and cooperative effects of dissipation and driving were reported [86]. However, a more thorough understanding of the nature of non-Markovianity was required to understand its interplay with quantum control. As described in Sec. II A, non-Markovianity has in the meantime been characterized in terms of information flow from the environment to the system [75], increase of correlations in a bipartite system [73], or re-expansion of the volume of ac- 
cessible states [78], among others. The important point in the context of quantum control is that each of these measures holds a promise for better control: Correlations between system and environment may improve fidelities of single qubit gates [38], cooperative effects of control and dissipation may allow for entropy export and thus cooling [86, 149]; and harnessing non-Markovianity may enhance the efficiency of quantum information processing and communication [79, 194].

A first example of exploiting non-Markovianity for quantum control was reported in Ref. [70], showing that, for an anharmonic ladder system, the environment may be utilized to extend possible operations from $S O(N)$ to $S U(N)$. Presence of at least one two-level defect in the environment that is sufficiently isolated and sufficiently strongly coupled to the system was identified as prerequisite for the observed controllability enhancement. Such conditions are found in current experiments with superconducting circuits and other systems which are immersed in a small, 'natural' spin bath, for example color centers in diamond.

The limited number of optimal control studies of open quantum systems with non-Markovian dynamics $[38,70$, $86,149,193]$ testifies to the fact that control of these systems remains largely uncharted territory. The full potential of the specific features of non-Markovian dynamics for quantum control remains yet to be explored. Open questions include, for example, how the build-up of memory influences control; whether specific features of the spectral density can be exploited for control, and if so, how. The tools for performing these studies, both in terms of simulating non-Markovian dynamics [61] and carrying out optimal control calculations, cf. Sec. IV A, have been developed and are there to be used.

\section{CONCLUSIONS}

The present review has been focused on control of open quantum systems as they are encountered in the field of quantum technologies. It will be concluded by briefly mentioning examples from other fields of current interest: Quantum optimal control for open systems has been employed in the context of quantum thermodynamics, in order to determine the optimal efficiency of a noisy heat engine [195]; biological chromophore complexes, in order to maximize exciton transfer [196, 197]; molecules immersed in dissipative media, in order to maximally align them with respect to a laboratory axis [198-200]; molecular junctions, in order to control the current, shot noise and Fano factors [201]; as well as chemical reaction dynamics [202-204], including charge transfer in molecules [205], and surface photochemistry [206-208]. The numerous applications attest to the maturity as well as versatility of the quantum control toolbox [1].

At this stage, three rules for controlling open quantum systems may tentatively be formulated:

1. If the desired operation shall keep pure states pure,
Markovian dynamics are unwanted. The effect of the environment in this case is detrimental. A suitable strategy is then to perform any desired operation as fast as possible. Quantum optimal control theory is a viable tool to determine both the shortest operation time and the control that drives the desired dynamics. The actual dissipative processes may be neglected during the optimization for computational simplicity. Explicit account of the dissipative processes comes at a significantly larger numerical cost but allows for identifying subspaces which are less affected by or even immune to decoherence.

2. If the desired operation changes the purity of the system's state, presence of the environment is necessary for realizing the control target. In this case, Markovian dynamics may be desired: The control target is reachable if it is a fixed state of the Liouvillian. External control fields may be used to ensure that this is the case. The corresponding control strategy is referred to as quantum reservoir engineering. If the Liouvillian has several fixed points, external fields may also be used to drive the dynamics to the desired one. The role of non-Markovian effects in this type of desired dissipation has not been explored to date.

3. Non-Markovian dynamics in general may have both beneficial and detrimental effects on controlled quantum dynamics. Improved controllability is a first example of a benefit. It requires presence of a few strongly coupled and sufficiently isolated environmental modes which can effectively act as ancillas [212], and use of quantum optimal control for properly exploiting these modes. An improved quantum speed limit is a second example.

While the number of examples for successful control of open quantum systems is growing, our current understanding of controllability and the most promising control strategies for open quantum systems is still rather limited. In particular, a thorough understanding of the role of non-Markovian effects is lacking to date, and it is currently still unknown which features of nonMarkovianity can be exploited for quantum control.

Investigation of a larger range of models, with both small and large baths, consisting of harmonic modes and spins, and a systematic analysis of non-Markovianity may elucidate this question. Such an improved understanding would not only be crucial for advancing quantum technologies but would also be beneficial for adjacent fields such as condensed matter physics or chemical reaction dynamics.

\section{Acknowledgments}

I would like to thank Ugo Boscain, Steffen Glaser, Dominique Sugny, and David Tannor for their comments on 
the manuscript. Recent advances in the control of open quantum systems in my own group were made possible by the work of Michael Goerz, Giulia Gualdi and Daniel
Reich whom I would like to thank for their dedication and perserverance.
[1] S. J. Glaser, U. Boscain, T. Calarco, C. P. Koch, W. Köckenberger, R. Kosloff, I. Kuprov, B. Luy, S. Schirmer, T. Schulte-Herbrüggen, et al., Eur. Phys. J. D 69 (2015).

[2] D. D'Alessandro, Introduction to Quantum Control and Dynamics (Chapman \& Hall/CRC, 2007).

[3] M. Shapiro and P. Brumer, Quantum Control of Molecular Processes (Wiley Interscience, 2012), 2nd ed.

[4] L. S. Pontryagin, V. G. Boltyanskii, R. V. Gamkrelidze, and E. F. Mishchenko, The Mathematical Theory of $O p$ timal Processes (Wiley, New York, 1962).

[5] V. F. Krotov, Global Methods in Optimal Control (Marcel Dekker, New York, 1996).

[6] S. Conolly, D. Nishimura, and A. Macovski, IEEE Trans. Med. Imaging MI-5, 106 (1986).

[7] J. Mao, T. H. Mareci, K. N. Scott, and E. Andrew, J. Magn. Reson. 70, 310 (1986).

[8] D. Tannor and S. Rice, J. Chem. Phys. 83, 5013 (1985).

[9] A. P. Peirce, M. A. Dahleh, and H. Rabitz, Phys. Rev. A 37, 4950 (1988).

[10] R. Kosloff, S. Rice, P. Gaspard, S. Tersigni, and D. Tannor, Chem. Phys. 139, 201 (1989).

[11] D. Tannor, V. Kazakov, and V. Orlov, in Timedependent quantum molecular dynamics, edited by J. Broeckhove and L. Lathouwers (Plenum, 1992), pp. 347-360.

[12] P. Gross, D. Neuhauser, and H. Rabitz, J. Chem. Phys. 96, 2834 (1992).

[13] J. Somlói, V. A. Kazakovski, and D. J. Tannor, Chem. Phys. 172, 85 (1993).

[14] A. Assion, T. Baumert, X. Bergt, T. Brixner, X. Kiefer, V. Seyfried, X. Strehle, and G. Gerber, Science 282, 919 (1998).

[15] R. S. Judson and H. Rabitz, Phys. Rev. Lett. 68, 1500 (1992).

[16] B. Schäfer-Bung, R. Mitrić, V. Bonačić-Koutecký, A. Bartelt, C. Lupulescu, A. Lindinger, v. Vajda, S. M. Weber, and L. Wöste, J. Phys. Chem. A 108, 4175 (2004).

[17] W. Zhu, J. Botina, and H. Rabitz, J. Chem. Phys. 108, 1953 (1998).

[18] A. M. Weiner, Rev. Sci. Instrum. 71, 1929 (2000).

[19] M. J. Wright, P. L. Gould, and S. D. Gensemer, Rev. Sci. Instrum. 75, 4718 (2004).

[20] F. Motzoi, J. M. Gambetta, S. T. Merkel, and F. K. Wilhelm, Phys. Rev. A 84, 022307 (2011).

[21] G. Jäger and U. Hohenester, Phys. Rev. A 88, 035601 (2013).

[22] K. Kobzar, B. Luy, N. Khaneja, and S. J. Glaser, J. Magn. Reson. 173, 229 (2005).

[23] T. E. Skinner, K. Kobzar, B. Luy, M. R. Bendall, W. Bermel, N. Khaneja, and S. J. Glaser, J. Magn. Reson. 179, 241 (2006).

[24] M. Nimbalkar, B. Luy, T. E. Skinner, J. L. Neves, N. I. Gershenzon, K. Kobzar, W. Bermel, and S. J. Glaser, J. Magn. Reson. 228, 16 (2013).
[25] J. P. Palao and R. Kosloff, Phys. Rev. Lett. 89, 188301 (2002).

[26] C. Tesch and R. de Vivie-Riedle, Phys. Rev. Lett. 89, 157901 (2002).

[27] J. P. Palao and R. Kosloff, Phys. Rev. A 68, 062308 (2003).

[28] F. Platzer, F. Mintert, and A. Buchleitner, Phys. Rev. Lett. 105, 020501 (2010).

[29] M. M. Müller, D. M. Reich, M. Murphy, H. Yuan, J. Vala, K. B. Whaley, T. Calarco, and C. P. Koch, Phys. Rev. A 84, 042315 (2011).

[30] T. Caneva, T. Calarco, and S. Montangero, New J. Phys. 14, 093041 (2012).

[31] D. J. Egger and F. K. Wilhelm, Phys. Rev. A 90, 052331 (2014).

[32] U. V. Poulsen, S. Sklarz, D. Tannor, and T. Calarco, Phys. Rev. A 82, 012339 (2010).

[33] P. Treutlein, T. W. Hänsch, J. Reichel, A. Negretti, M. A. Cirone, and T. Calarco, Phys. Rev. A 74, 022312 (2006).

[34] M. H. Goerz, T. Calarco, and C. P. Koch, J. Phys. B 44, 154011 (2011).

[35] M. H. Goerz, E. J. Halperin, J. M. Aytac, C. P. Koch, and K. B. Whaley, Phys. Rev. A 90, 032329 (2014).

[36] J. Scheuer, X. Kong, R. S. Said, J. Chen, A. Kurz, L. Marseglia, J. Du, P. R. Hemmer, S. Montangero, T. Calarco, et al., New J. Phys. 16, 093022 (2014).

[37] G. Waldherr, Y. Wang, S. Zaiser, M. Jamali, T. SchulteHerbrüggen, H. Abe, T. Ohshima, J. Isoya, J. F. Du, P. Neumann, et al., Nature 506, 204 (2014).

[38] P. Rebentrost, I. Serban, T. Schulte-Herbrüggen, and F. K. Wilhelm, Phys. Rev. Lett. 102, 090401 (2009).

[39] M. H. Goerz, D. M. Reich, and C. P. Koch., New J. Phys. 16, 055012 (2014).

[40] J. Grond, J. Schmiedmayer, and U. Hohenester, Phys. Rev. A 79, 021603 (2009).

[41] X. Wang, A. Bayat, S. Bose, and S. G. Schirmer, Phys. Rev. A 82, 012330 (2010).

[42] S. Machnes, U. Sander, S. J. Glaser, P. de Fouquières, A. Gruslys, S. Schirmer, and T. Schulte-Herbrüggen, Phys. Rev. A 84, 022305 (2011).

[43] P. Doria, T. Calarco, and S. Montangero, Phys. Rev. Lett. 106, 190501 (2011).

[44] K. Rojan, D. M. Reich, I. Dotsenko, J.-M. Raimond, C. P. Koch, and G. Morigi, Phys. Rev. A 90, 023824 (2014).

[45] G. De Chiara, T. Calarco, M. Anderlini, S. Montangero, P. J. Lee, B. L. Brown, W. D. Phillips, and J. V. Porto, Phys. Rev. A 77, 052333 (2008).

[46] T. Caneva, M. Murphy, T. Calarco, R. Fazio, S. Montangero, V. Giovannetti, and G. E. Santoro, Phys. Rev. Lett. 103, 240501 (2009).

[47] H. A. Fürst, M. H. Goerz, U. G. Poschinger, M. Murphy, S. Montangero, T. Calarco, F. Schmidt-Kaler, K. Singer, and C. P. Koch., New J. Phys. 16, 075007 (2014). 
[48] A. V. Gorshkov, T. Calarco, M. D. Lukin, and A. S. Sørensen, Phys. Rev. A 77, 043806 (2008).

[49] S. Rosi, A. Bernard, N. Fabbri, L. Fallani, C. Fort, M. Inguscio, T. Calarco, and S. Montangero, Phys. Rev. A 88, 021601 (2013).

[50] S. van Frank, M. Bonneau, J. Schmiedmayer, S. Hild, C. Gross, M. Cheneau, I. Bloch, T. Pichler, A. Negretti, T. Calarco, et al., arXiv:1511.02247 (2015).

[51] T. Häberle, D. Schmid-Lorch, K. Karrai, F. Reinhard, and J. Wrachtrup, Phys. Rev. Lett. 111, 170801 (2013).

[52] F. Dolde, V. Bergholm, Y. Wang, I. Jakobi, B. Naydenov, S. Pezzagna, J. Meijer, F. Jelezko, P. Neumann, T. Schulte-Herbrüggen, et al., Nat. Commun. 5, 3371 (2014).

[53] S. van Frank, A. Negretti, T. Berrada, R. Bücker, S. Montangero, J.-F. Schaff, T. Schumm, T. Calarco, and J. Schmiedmayer, Nat. Commun. 5, 4009 (2014).

[54] U. Weiss, Quantum dissipative systems (World Scientific, Singapore, 2012), 4th ed.

[55] H.-P. Breuer and F. Petruccione, The theory of open quantum systems (Oxford University Press, 2007).

[56] H.-P. Breuer, E.-M. Laine, J. Piilo, and B. Vacchini, arXiv:1505.01385 (2015).

[57] A. Rivas, S. F. Huelga, and M. B. Plenio, Rep. Prog. Phys. 77, 094001 (2014).

[58] R. Kosloff, Annu. Rev. Phys. Chem. 45, 145 (1994).

[59] F. Lucas and K. Hornberger, Phys. Rev. A 89, 012112 (2014).

[60] R. Sweke, I. Sinayskiy, D. Bernard, and F. Petruccione, Phys. Rev. A 91, 062308 (2015).

[61] I. de Vega and D. Alonso, arXiv:1511.06994 (2015).

[62] J. Piilo, S. Maniscalco, K. Härkönen, and K.-A. Suominen, Phys. Rev. Lett. 100, 180402 (2008).

[63] W. Koch, F. Großmann, J. T. Stockburger, and J. Ankerhold, Phys. Rev. Lett. 100, 230402 (2008).

[64] L. Diósi and L. Ferialdi, Phys. Rev. Lett. 113, 200403 (2014).

[65] C. Meier and D. J. Tannor, J. Chem. Phys. 111, 3365 (1999).

[66] C. P. Koch, T. Klüner, H.-J. Freund, and R. Kosloff, Phys. Rev. Lett. 90, 117601 (2003).

[67] K. H. Hughes, C. D. Christ, and I. Burghardt, J. Chem. Phys. 131, 024109 (2009).

[68] I. M. Georgescu, S. Ashhab, and F. Nori, Rev. Mod. Phys. 86, 153 (2014).

[69] G. Gualdi and C. P. Koch, Phys. Rev. A 88, 022122 (2013).

[70] D. M. Reich, N. Katz, and C. P. Koch, Sci. Rep. 5, 12430 (2015).

[71] G. Katz, D. Gelman, M. A. Ratner, and R. Kosloff, J. Chem. Phys. 129, 034108 (2008).

[72] M. M. Wolf, J. Eisert, T. S. Cubitt, and J. I. Cirac, Phys. Rev. Lett. 101, 150402 (2008).

[73] A. Rivas, S. F. Huelga, and M. B. Plenio, Phys. Rev. Lett. 105, 050403 (2010).

[74] H. S. Dhar, M. N. Bera, and G. Adesso, Phys. Rev. A 91, 032115 (2015).

[75] H.-P. Breuer, E.-M. Laine, and J. Piilo, Phys. Rev. Lett. 103, 210401 (2009).

[76] E.-M. Laine, J. Piilo, and H.-P. Breuer, Phys. Rev. A 81, 062115 (2010).

[77] R. Vasile, S. Maniscalco, M. G. A. Paris, H.-P. Breuer, and J. Piilo, Phys. Rev. A 84, 052118 (2011).

[78] S. Lorenzo, F. Plastina, and M. Paternostro, Phys. Rev.
A 88, 020102 (2013).

[79] B. Bylicka, D. Chruściński, and S. Maniscalco, Sci. Rep. 4, 5720 (2014).

[80] C. Addis, B. Bylicka, D. Chruściński, and S. Maniscalco, Phys. Rev. A 90, 052103 (2014).

[81] H.-B. Chen, J.-Y. Lien, G.-Y. Chen, and Y.-N. Chen, Phys. Rev. A 92, 042105 (2015).

[82] J. Bae and D. Chruscinski, arXiv:1601.05522 (2016).

[83] B.-H. Liu, L. Li, Y.-F. Huang, C.-F. Li, G.-C. Guo, E.M. Laine, H.-P. Breuer, and J. Piilo, Nature Phys. 7, 931 (2011).

[84] M. Gessner, M. Ramm, T. Pruttivarasin, A. Buchleitner, H.-P. Breuer, and H. Häffner, Nature Phys. 10, 105 (2014).

[85] A. Bartana, R. Kosloff, and D. J. Tannor, J. Chem. Phys. 106, 1435 (1997).

[86] R. Schmidt, A. Negretti, J. Ankerhold, T. Calarco, and J. T. Stockburger, Phys. Rev. Lett. 107, 130404 (2011).

[87] M. Viteau, A. Chotia, M. Allegrini, N. Bouloufa, O. Dulieu, D. Comparat, and P. Pillet, Science 321, 232 (2008).

[88] D. M. Reich and C. P. Koch, New J. Phys. 15, 125028 (2013).

[89] S. Kallush and R. Kosloff, Phys. Rev. A 73, 032324 (2006).

[90] T. Schulte-Herbrüggen, A. Spörl, N. Khaneja, and S. J. Glaser, J. Phys. B 44, 154013 (2011).

[91] A. Bendersky, F. Pastawski, and J. P. Paz, Phys. Rev. Lett. 100, 190403 (2008).

[92] M. P. da Silva, O. Landon-Cardinal, and D. Poulin, Phys. Rev. Lett. 107, 210404 (2011).

[93] S. T. Flammia and Y.-K. Liu, Phys. Rev. Lett. 106, 230501 (2011).

[94] E. Magesan, J. M. Gambetta, and J. Emerson, Phys. Rev. Lett. 106, 180504 (2011).

[95] D. M. Reich, G. Gualdi, and C. P. Koch, Phys. Rev. Lett. 111, 200401 (2013).

[96] D. M. Reich, G. Gualdi, and C. P. Koch, Phys. Rev. A 88, 042309 (2013).

[97] D. M. Reich, M. Ndong, and C. P. Koch, J. Chem. Phys. 136, 104103 (2012).

[98] P. Watts, J. Vala, M. M. Müller, T. Calarco, K. B. Whaley, D. M. Reich, M. H. Goerz, and C. P. Koch, Phys. Rev. A 91, 062306 (2015).

[99] M. H. Goerz, G. Gualdi, D. M. Reich, C. P. Koch, F. Motzoi, K. B. Whaley, J. Vala, M. M. Müller, S. Montangero, and T. Calarco, Phys. Rev. A 91, 062307 (2015).

[100] D. M. Reich, Ph.D. thesis, Universität Kassel (2015), URL http://nbn-resolving.de/urn:nbn: de:hebis:34-2015061948576.

[101] V. Jurdjevic, Geometric Control Theory, Cambridge Studies in Advanced Mathematics (Cambridge Univ. Press, Cambridge, 1997).

[102] V. Jurdjevic and H. J. Sussmann, J. Diff. Eqns. 12, 313 (1972), ISSN 0022-0396.

[103] G. M. Huang, T. J. Tarn, and C. W. Clark, J. Math. Phys. 24, 2608 (1983).

[104] T. Polack, H. Suchowski, and D. J. Tannor, Phys. Rev. A 79, 053403 (2009).

[105] C. Arenz, G. Gualdi, and D. Burgarth, New J. Phys. 16, 065023 (2014).

[106] R. Wu, A. Pechen, C. Brif, and H. Rabitz, J. Phys. A 40, 5681 (2007). 
[107] F. Ticozzi and L. Viola, Sci. Rep. 4, 5192 (2014).

[108] T. Chambrion, P. Mason, M. Sigalotti, and U. Boscain, Ann. Inst. Henri Poincaré 26, 329 (2009).

[109] K. Beauchard, J.-M. Coron, and P. Rouchon, Commun. Math. Phys. 296, 525 (2010).

[110] N. Boussaid, M. Caponigro, and T. Chambrion, IEEE Trans. Automat. Control 58, 2205 (2013).

[111] U. Boscain, J.-P. Gauthier, F. Rossi, and M. Sigalotti, Commun. Math. Phys. 333, 1225 (2015), ISSN 00103616.

[112] C. Altafini, J. Math. Phys. 44, 2357 (2003).

[113] S. Lloyd and L. Viola, Phys. Rev. A 65, 010101 (2001).

[114] G. Dirr, U. Helmke, I. Kurniawan, and T. SchulteHerbrüggen, Rep. Math. Phys. 64, 93 (2009).

[115] C. O'Meara, G. Dirr, and T. Schulte-Herbruggen, IEEE Trans. Automatic Control 57, 2050 (2012), ISSN 00189286.

[116] C. Altafini and F. Ticozzi, IEEE Trans. Automatic Control 57, 1898 (2012), ISSN 0018-9286.

[117] B. Dive, D. Burgarth, and F. Mintert, arXiv:1509.07163 (2015).

[118] D. A. Lidar, I. L. Chuang, and K. B. Whaley, Phys. Rev. Lett. 81, 2594 (1998).

[119] D. A. Lidar and K. B. Whaley, in Irreversible Quantum Dynamics, edited by F. Benatti and R. Floreani (Berlin, 2003), vol. 622 of Springer Lecture Notes in Physics, pp. $83-120$.

[120] L.-M. Duan and G.-C. Guo, Phys. Rev. Lett. 79, 1953 (1997).

[121] E. M. Fortunato, L. Viola, J. Hodges, G. Teklemariam, and D. G. Cory, New J. Phys. 4, 5 (2002).

[122] T. Monz, K. Kim, A. S. Villar, P. Schindler, M. Chwalla, M. Riebe, C. F. Roos, H. Häffner, W. Hänsel, M. Hennrich, et al., Phys. Rev. Lett. 103, 200503 (2009).

[123] X. Wang, M. Byrd, and K. Jacobs, Phys. Rev. A 87, $012338(2013)$.

[124] X. X. Yi, X. L. Huang, C. Wu, and C. H. Oh, Phys. Rev. A 80, 052316 (2009).

[125] E. Knill, R. Laflamme, and L. Viola, Phys. Rev. Lett. 84, 2525 (2000).

[126] L. Viola, E. M. Fortunato, M. A. Pravia, E. Knill, R. Laflamme, and D. G. Cory, Science 293, 2059 (2001).

[127] J. S. Waugh, Average Hamiltonian Theory (John Wiley \& Sons, Ltd., 2007).

[128] L. Viola, E. Knill, and S. Lloyd, Phys. Rev. Lett. 82, 2417 (1999)

[129] L. Viola, J. Mod. Opt. 51, 2357 (2004).

[130] A. M. Souza, G. A. Álvarez, and D. Suter, Phil. Trans. R. Soc. A 370, 4748 (2012), ISSN 1364-503X.

[131] D. Suter and G. A. Álvarez, Rev. Mod. Phys. p. in press (2016).

[132] K. Khodjasteh and D. A. Lidar, Phys. Rev. Lett. 95, 180501 (2005).

[133] M. J. Biercuk, H. Uys, A. P. VanDevender, N. Shiga, W. M. Itano, and J. J. Bollinger, Nature 458, 996 (2009).

[134] J. Bylander, S. Gustavsson, F. Yan, F. Yoshihara, K. Harrabi, G. Fitch, D. G. Cory, Y. Nakamura, J.-S. Tsai, and W. D. Oliver, Nature Phys. 7, 565570 (2011).

[135] T. Green, H. Uys, and M. J. Biercuk, Phys. Rev. Lett. 109, 020501 (2012).

[136] G. A. Paz-Silva and L. Viola, Phys. Rev. Lett. 113, 250501 (2014).
[137] A. Soare, H. Ball, D. Hayes, J. Sastrawan, M. C. Jarratt, J. J. McLoughlin, X. Zhen, T. J. Green, and M. J. Biercuk, Nature Phys. 10, 825 (2014).

[138] J. Clausen, G. Bensky, and G. Kurizki, Phys. Rev. Lett. 104, 040401 (2010).

[139] O. Viyuela, A. Rivas, and M. A. Martin-Delgado, Phys. Rev. B 86, 155140 (2012).

[140] A. Rivas, O. Viyuela, and M. A. Martin-Delgado, Phys. Rev. B 88, 155141 (2013).

[141] L. Cywiński, R. M. Lutchyn, C. P. Nave, and S. Das Sarma, Phys. Rev. B 77, 174509 (2008).

[142] S. Kotler, N. Akerman, Y. Glickman, A. Keselman, and R. Ozeri, Nature 473, 61 (2011).

[143] S. Kotler, N. Akerman, Y. Glickman, and R. Ozeri, Phys. Rev. Lett. 110, 110503 (2013).

[144] N. Khaneja, B. Luy, and S. J. Glaser, Proc. Natl. Acad. Sci. USA 100, 13162 (2003).

[145] N. Khaneja, T. Reiss, B. Luy, and S. J. Glaser, J. Magn. Reson. 162, 311 (2003).

[146] M. Lapert, Y. Zhang, M. Braun, S. J. Glaser, and D. Sugny, Phys. Rev. Lett. 104, 083001 (2010).

[147] M. Lapert, E. Assémat, S. J. Glaser, and D. Sugny, Phys. Rev. A 88, 033407 (2013).

[148] V. Mukherjee, A. Carlini, A. Mari, T. Caneva, S. Montangero, T. Calarco, R. Fazio, and V. Giovannetti, Phys. Rev. A 88, 062326 (2013).

[149] V. Mukherjee, V. Giovannetti, R. Fazio, S. F. Huelga, T. Calarco, and S. Montangero, New J. Phys. 17, 063031 (2015).

[150] Y. Rezek, P. Salamon, K. H. Hoffmann, and R. Kosloff, Europhys. Lett. 85, 30008 (2009).

[151] K. H. Hoffmann, P. Salamon, Y. Rezek, and R. Kosloff, Europhys. Lett. 96, 60015 (2011)

[152] S. E. Sklarz, D. J. Tannor, and N. Khaneja, Phys. Rev. A 69, 053408 (2004).

[153] H. Yuan, C. P. Koch, P. Salamon, and D. J. Tannor, Phys. Rev. A 85, 033417 (2012).

[154] A. Bartana, R. Kosloff, and D. J. Tannor, J. Chem. Phys. 99, 196 (1993).

[155] D. J. Tannor and A. Bartana, J. Phys. Chem. A 103, 10359 (1999).

[156] R. Schmidt, S. Rohrer, J. Ankerhold, and J. T. Stockburger, Physica Scripta 2012, 014034 (2012).

[157] Y. Ohtsuki, W. Zhu, and H. Rabitz, J. Chem. Phys. 110, 9825 (1999).

[158] N. Khaneja, T. Reiss, C. Kehlet, T. SchulteHerbrüggen, and S. J. Glaser, J. Magn. Reson. 172, 296 (2005).

[159] K. Sundermann and R. d. Vivie-Riedle, J. Chem. Phys. 110, 1896 (1999).

[160] R. Eitan, M. Mundt, and D. J. Tannor, Phys. Rev. A 83, 053426 (2011).

[161] M. H. Goerz, K. B. Whaley, and C. P. Koch, EPJ Quantum Technology 2, 21 (2015), ISSN 2196-0763.

[162] J. P. Palao, D. M. Reich, and C. P. Koch, Phys. Rev. A 88, 053409 (2013).

[163] D. M. Reich, J. P. Palao, and C. P. Koch, J. Mod. Opt. 61, 822 (2014).

[164] R. E. Goetz, A. Karamatskou, R. Santra, and C. P. Koch, Phys. Rev. A 93, 013413 (2016).

[165] K. W. Moore and H. Rabitz, J. Chem. Phys. 137, 134113 (2012).

[166] G. Riviello, K. Moore Tibbetts, C. Brif, R. Long, R.-B. Wu, T.-S. Ho, and H. Rabitz, Phys. Rev. A 91, 043401 
(2015).

[167] D. J. Gorman, K. C. Young, and K. B. Whaley, Phys. Rev. A 86, 012317 (2012).

[168] G. Quiroz and D. A. Lidar, Phys. Rev. A 88, 052306 (2013).

[169] G. A. Paz-Silva and D. A. Lidar, Sci. Rep. 3, 1530 (2013).

[170] G. Katz, M. A. Ratner, and R. Kosloff, Phys. Rev. Lett. 98, 203006 (2007).

[171] D. Stefanatos, N. Khaneja, and S. J. Glaser, Phys. Rev. A 69, 022319 (2004).

[172] N. I. Gershenzon, K. Kobzar, B. Luy, S. J. Glaser, and T. E. Skinner, J. Magn. Reson. 188, 330 (2007).

[173] V. Giovannetti, S. Lloyd, and L. Maccone, Phys. Rev. A 67, 052109 (2003).

[174] L. B. Levitin and T. Toffoli, Phys. Rev. Lett. 103, 160502 (2009).

[175] G. C. Hegerfeldt, Phys. Rev. Lett. 111, 260501 (2013).

[176] M. H. Goerz, Ph.D. thesis, Universität Kassel (2015), URL http://nbn-resolving.de/urn:nbn: de:hebis:34-20150

[177] U. Hohenester and G. Stadler, Phys. Rev. Lett. 92, 196801 (2004).

[178] U. Hohenester, J. Phys. B 40, S315 (2007).

[179] S. Deffner and E. Lutz, Phys. Rev. Lett. 111, 010402 (2013).

[180] A. D. Cimmarusti, Z. Yan, B. D. Patterson, L. P. Corcos, L. A. Orozco, and S. Deffner, Phys. Rev. Lett. 114, $233602(2015)$.

[181] S. Machnes, J. Cerrillo, M. Aspelmeyer, W. Wieczorek, M. B. Plenio, and A. Retzker, Phys. Rev. Lett. 108, 153601 (2012).

[182] A. Rahmani, T. Kitagawa, E. Demler, and C. Chamon, Phys. Rev. A 87, 043607 (2013).

[183] J. F. Poyatos, J. I. Cirac, and P. Zoller, Phys. Rev. Lett. 77, 4728 (1996).

[184] S. Pielawa, G. Morigi, D. Vitali, and L. Davidovich, Phys. Rev. Lett. 98, 240401 (2007).

[185] S. Diehl, A. Micheli, A. Kantian, B. Kraus, H.-P. Büchler, and P. Zoller, Nature Phys. 4, 878 (2008).

[186] S. G. Schirmer and X. Wang, Phys. Rev. A 81, 062306 (2010).

[187] F. Motzoi, E. Halperin, X. Wang, K. B. Whaley, and S. Schirmer, arXiv:1512.03415 (2015).

[188] A. Pechen and H. Rabitz, Phys. Rev. A 73, 062102 (2006).

[189] A. Pechen, N. Il'in, F. Shuang, and H. Rabitz, Phys. Rev. A 74, 052102 (2006).

[190] D. K. Burgarth, P. Facchi, V. Giovannetti, H. Nakazato, S. Pascazio, and K. Yuasa, Nature Commun. 5, 5173 (2014).

[191] C. Arenz, D. Burgarth, P. Facchi, V. Giovan- netti, H. Nakazato, S. Pascazio, and K. Yuasa, arXiv:1601.01212 (2016)

[192] F. Shuang, A. Pechen, T.-S. Ho, and H. Rabitz, J. Chem. Phys. 126, 134303 (2007).

[193] F. F. Floether, P. de Fouquieres, and S. G. Schirmer, New J. Phys. 14, 073023 (2012).

[194] E.-M. Laine, H.-P. Breuer, and J. Piilo, Sci. Rep. 4, 4620 (2014).

[195] D. Stefanatos, Phys. Rev. E 90, 012119 (2014).

[196] B. Brüggemann, T. Pullerits, and V. May, J. Photochem. Photobiol. A 190, 372 (2007).

[197] S. Hoyer, F. Caruso, S. Montangero, M. Sarovar, T. Calarco, M. B. Plenio, and K. B. Whaley, New J. Phys. 16, 045007 (2014).

[198] A. Pelzer, S. Ramakrishna, and T. Seideman, J. Chem. Phys. 129, 134301 (2008).

[199] D. Zhdanov and H. Rabitz, Phys. Rev. A 83, 061402 (2011).

[200] T. Vieillard, F. Chaussard, F. Billard, D. Sugny, O. Faucher, S. Ivanov, J.-M. Hartmann, C. Boulet, and 274838B. Lavorel, Phys. Rev. A 87, 023409 (2013).

[201] G.-Q. Li and U. Kleinekathöfer, Eur. Phys. J. B 76, 309 (2010).

[202] D. Sugny, M. Ndong, D. Lauvergnat, Y. Justum, and M. Desouter-Lecomte, J. Photochem. Photobiol. A 190, 359 (2007), ISSN 1010-6030, theoretical Aspects of Photoinduced Processes in Complex Systems.

[203] A. Chenel, G. Dive, C. Meier, and M. DesouterLecomte, J. Phys. Chem. A 116, 11273 (2012).

[204] A. Chenel, C. Meier, G. Dive, and M. DesouterLecomte, J. Chem. Phys. 142, 024307 (2015).

[205] J. C. Tremblay and P. Saalfrank, Phys. Rev. A 78, 063408 (2008).

[206] K. Mishima and K. Yamashita, J. Chem. Phys. 131, 014109 (2009).

[207] E. Asplund and T. Klüner, Phys. Rev. Lett. 106, 140404 (2011).

[208] J. C. Tremblay, G. Füchsel, and P. Saalfrank, Phys. Rev. B 86, 045438 (2012).

[209] G. Gualdi, D. Licht, D. M. Reich, and C. P. Koch, Phys. Rev. A 90, 032317 (2014).

[210] D. M. Reich, G. Gualdi, and C. P. Koch, J. Phys. A: Math. Theor. 47, 385305 (2014).

[211] The problem is slightly more involved if one seeks an estimate of the error instead of a simple yes-no answer. It is addressed in Refs. [95, 209, 210]. For control applications, however, Eq. (3) and variants are sufficient[39].

[212] An important difference to true ancilla systems is controllability: Environmental modes are by definition uncontrollable and can only be accessed via their coupling to the system. 